\title{
COMMUNICATION
}

\section{Conséquences dentaires de la dysjonction pterygo-maxillaire pendant les chirugies d'avancée fronto-faciale monobloc dans le traitement des syndromes de Crouzon}

\author{
L. Sicard (Paris), R. Khonsari (Paris)
}

Le syndrome de Crouzon est une faciocraniosténose qui peut s'exprimer par une retrusion fronto-faciale sévère associée à des répercussions fonctionnelles importantes : un exorbitisme sévère peut mener à une mise en jeux du pronostic visuel et les troubles de la ventilation mettre en cause le pronostic vital. La procédure de choix du traitement primaire de cette faciocraniosténose est l'avancée monobloc frontofaciale avec distraction par voie interne ou externe. Cette technique nécessite la réalisation d'une disjonction ptérygo-maillaire, pratiquée par voie supérieure ou intra-orale. Cette disjonction est associée à un risque de lésion des germes des dents temporaires et permanentes. 15 patients atteints du syndromes de Crouzon, opérés par avancée monobloc fronto-faciale, par le même chirurgien et avec disjonction pterygo-maxillaire par voie supérieure en passant par la fosse temporale, ont été aléatoirement choisis parmi les patients atteint du syndrome de Crouzon suivis dans le service. La formule dentaire de chaque patient a été relevée sur les scanners pré-opératoires, post-opératoires et de contrôle, ainsi que les différentes anomalies et stades de Nolla des premières et deuxièmes molaires permanentes maxillaires. L'étude confirme que cette technique chirurgicale cause des dommages dentaires notables et montre que la survenues de ces dommages est corrélée à l'âge auquel est réalisée la chirurgie. Elle montre que l'existence de dommage au niveau de la première molaire permanente est un facteur prédictif de séquelles au niveau de la 2 ème molaire permanente lors de son évolution. Le stade radiographique de Nolla peut être un indicateur plus fiable que l'âge civil pour évaluer les risques dentaire lors de cette chirurgie chez ces patients. Si les indications de chirurgie précoce par avancée fronto- faciale monobloc chez les patients atteints d'un syndrome de Crouzon avec répercussion fonctionnelles sévère ne se discutent pas, il sera intéressant de comparer ces résultats avec les séquelles dentaires de cette chirurgie réalisée avec disjonction ptérygo-palatine par voie endobuccale. 